Duvall et al. argue that it may be better for finding sound-speed stratification than the $\omega-k$ diagram.

What can we learn from this? Already, Duvall et al. have solved a puzzle about the presence of highpersist above $5,000 \mu \mathrm{Hz}$ (period around 3 minutes), the expected acoustical cutoff, but is this because of interference effects $^{4}$ or because of anomalously strong reflection at the solar surface ${ }^{5}$ ? The inversion by Duvall et al. shows that the paths of these high-frequency waves make only one return to the surface, which thus seems too diffuse to make a good reflecting boundary for them.

At present, however, the main result is the demonstration that a local analysis is even possible. Evaluation of the diagfrequency solar oscillations. Oscillations

nostic potential of the time-distance diagram probably requires simulations of the response of the observed correlation time and distance to various phenomena beneath the solar surface, such as flows and magnetic fields. But it seems likely that it will develop into a powerful aid to more traditional helioseismic techniques for probing the solar interior.

Jørgen Christensen-Dalsgaard is at the Institute of Physics and Astronomy, Aarhus University, DK-8000 Aarhus C, Denmark.

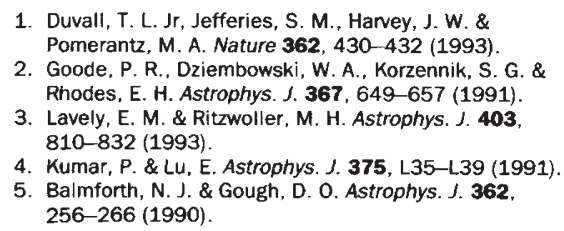

\section{That vaccine passes a trial}

\author{
F. E. G. Cox
}

ENCOURAGEMENT for those involved in the battle against malaria comes with publication of the results of a large, controlled human phase-III trial in Colombia, in which vaccinated individuals suffered fewer episodes of malaria over a one-year period than people in a placebo group ${ }^{1}$. The vaccine concerned has been the centre of contention for some years, as has its architect, the Colombian biochemist Manuel Patarroyo. His persistence now seems to have been vindicated.

Development of a malaria vaccine has become a major challenge, as insecticide and drug resistance have reduced the effectiveness of hitherto successful methods of control. But the task has proved a tough one because the parasite life-cycle involves a series of antigenically complex and diverse stages and the parasites have evolved ways, as yet undetermined, of evading the immune response. The possibilities of a vaccine are based on the observations that most of those infected do develop some degree of immunity, and encouraging results have been obtained in laboratory models. Of the different possible targets, the most favoured is the sporozoite, the infective stage injected by the mosquito vector. But vaccination trials have been equivocal and other targets now include the early liver stages, the blood stages that actually cause the disease, and even the stages in the mosquito taken up after the insect has fed on infected blood ${ }^{2}$.

Approaches to a vaccine have usually involved the systematic application of molecular techniques to identify putative protective antigens, and then to characterize and produce them in a recom- indicating that the vaccine would be suitable for Africa where the need for a childhood vaccine is greatest. From an immunological viewpoint, the most interesting findings are that there was no correlation between antibody levels and protection (meaning that cell-mediated mechanisms may be involved), that continual natural boosting was required to establish strong immunity, and that immunity was specific in that it did not extend to $P$. vivax.

On the face of it, the degree of protection achieved is not dramatic. But it is the first time that such a large trial has been undertaken and there can be no doubt that a certain degree of protection was achieved. This in itself justifies Patarroyo's approach, though it does not mean that the SPf66 is now the only candidate vaccine. It is now a basis for improvement by adding new components to it - as it is safe, and requires some experience of the infection for it to remain effective, one such component might be a transmission-blocking antigen effective only after the infected blood has been taken up by a mosquito ${ }^{6}$, or an anti-disease vaccine ${ }^{7}$, neither of which requires complete protection. In terms of control, mathematical models show that the incidence of malaria would be reduced by a drop in the rate of transmission, which would occur if those infected experienced fewer and less severe episodes of malaria. From the point of view of public health, fewer days would be lost by sickness if everyone were immunized with a vaccine, even only a partially effective one. Malaria is a complex disease, and it may be that all it will be possible to achieve in the near future will be a tolerable level of malaria with which it is possible to live.

SPf66 is being taken seriously by the World Health Organization and other trials are under way. In Ecuador, 193 people have been vaccinated with SPf66 in an areas of less intense transmission; although the results were encouraging, the numbers subsequently infected were so few that no firm conclusions can be drawn ${ }^{8}$. The big test will come when, in a joint trial, the Swiss Tropical Institute and the Tanzanian health authorities attempt to find out whether this vaccine protects children in Africa. The trial is now in progress - the results will not be known until July 1994, but they might well determine the future course of development of malaria vaccines.

F. E. G. Cox is in the Division of Life Sciences, King's College London, Campden Hill Road, London W8 7AH, UK.

\footnotetext{
1. Valero, M. V. et al. Lancet 341, 705-710 (1993).

2. Cox, F. E. G. Trends Biotechnol. 9, 389-394 (1991)

3. Patarroyo, M. E et al Nature 328, 629-632 (1987).

4. Patarroyo, M. E. et al. Nature 332, 158-161 (1988).

5. Patarroyo, M. E. et al. Nature 332, 158-161 (1988).

6. Kaslow, D. C. et al. Nature 333, 74-75 (1988).

7. Playfair, J. H. L., Taverne, J., Bate, C. A. W. \& de Souza,

P. Immun. Today 11, 25-27 (1990).

8. Sempertegui, F. et al. Vaccine (in the press).
} 